7. Fenton JE, Brazier H, De Souza A, Hughes JP, McShane DP. The accuracy of citation and quotation in otolaryngology/head and neck surgery journals. Clin Otolaryngol Allied Sci 2000;25:40-4

8. Porrino JA Jr, Tan V, Daluiski A. Misquotation of a commonly referenced hand surgery study. J Hand Surg [Am] 2008;33:2-7

9. Rastegar DA, Wolfe L. Experience, expertise, or specialty? uses and misuses of a reference. J Fam Pract 2002;51:168.

Available online October 9, 2009 doi:10.1016/j.jaci.2009.07.047

\section{ITGB2 mutation combined with deleted ring 21 chromosome in a child with leukocyte adhesion deficiency}

\section{To the Editor:}

Leukocyte adhesion deficiency type 1 (LAD-1, MIM:116920) is a rare, autosomal-recessive primary immunodeficiency disorder caused by defects in $I T G B 2$, a gene mapping on chromosome $21 \mathrm{q} 22.3^{1-3}$ and encoding for the $\beta 2$-integrins subunit, CD18. ${ }^{4,5}$ LAD-1 is characterized by extreme elevation of blood neutrophil counts, recurrent fungal and bacterial infections, slow wound healing, and dystrophic scars from skin injuries. The severity of symptoms is correlated with the level of CD11/CD18 expression. Generally, patients with less than $1 \%$ expression of CD11/CD18 are susceptible to frequent and life-threatening systemic infections and require hematopoietic stem cell transplantation (HSCT).

Ring 21 (r[21]), is a rare chromosomal abnormality ${ }^{6}$ whose classic phenotype includes hypertonia, prominent nasal bridge, downward slanting palpebral fissures, protuberant occiput, and large ears. Microcephaly, sparse curly hair, prominent forehead, long eye lashes, broad anteverted nasal tip, long philtrum, thin upper lip, small mouth, and retrognathia are also commonly seen. ${ }^{7,8}$ Other manifestations include the consistent features of prenatal and postnatal growth retardation, hematologic disorder, a distinctive facies, and cognitive impairment.

We report on a child with LAD-1, dysmorphic features, and growth retardation caused by a combination of a point mutation in $I T G B 2$ and a gross deletion resulting in $\mathrm{r}(21)$.

The patient is the only child of nonconsanguineous parents. Delivered at 36 weeks after labor induction for suspected intrauterine growth retardation, his birth weight was $1785 \mathrm{~g}(<3 \mathrm{rd}$ percentile), length $42 \mathrm{~cm}(<3$ rd percentile) and head circumference $30 \mathrm{~cm}(<3 \mathrm{rd}$ percentile). Perinatality was characterized by delayed umbilical cord separation. During workup for herniotomy, a subvalvular aortic stenosis was discovered, and postoperatively slow wound healing was noted. Because of persistent anemia and leukocytosis $\left(52 \times 10^{9} / \mathrm{L}\right)$, he was referred to our

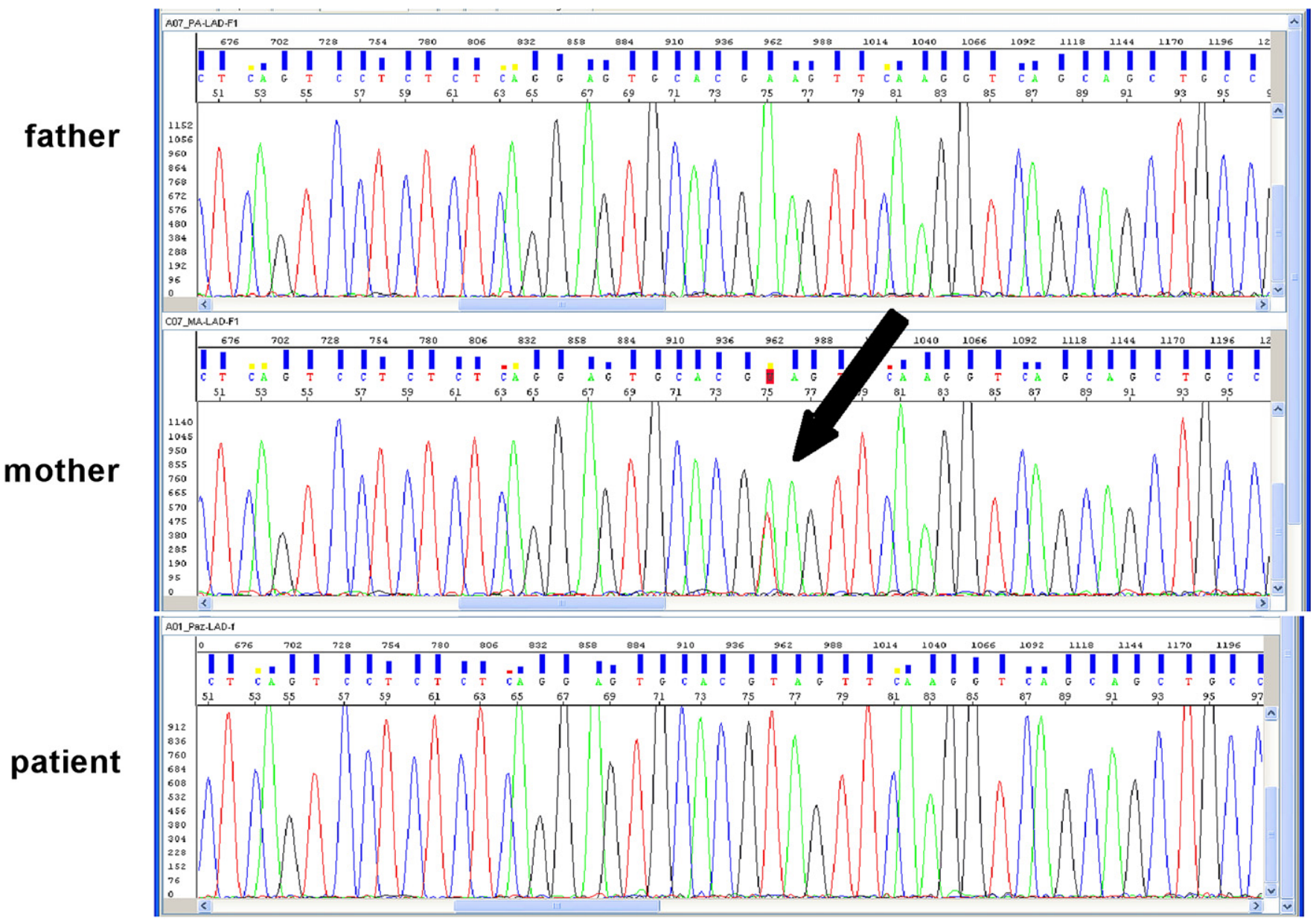

FIG 1. Chromatograms of portion of ITGB2 gene sequence in the proband and his parents. The c.79A $>T$ mutation is marked by an arrow. Note that for the mutated position, the father, the mother, and the affected son showed the AA, AT, and TT genotypes, respectively. 
center with a suspicion of primary immunodeficiency disorder. On admission, microcephaly, prominent forehead, flat nasal bridge, downward slanting palpebral fissures, and large ears were noted. He had sparse, thin hair, long eye lashes, broad anteverted nasal tip, long philtrum, a small mouth with thin lips, and micrognathia. A holosystolic murmur (4/6) was audible, and the hypoplastic scrotum harbored only the right testicle (volume $1 \mathrm{~mL}$ ). Swabs from the umbilicus and the surgical wound grew $E$ faecalis and $C$ parapsilosis. Immunologic workup revealed normal lymphocyte subpopulations with good proliferative response to mitogens, normal IgG and IgM, and low IgA serum levels. Lack of CD11b and CD18 expression on the surface of polymorphonuclear leukocytes and lymphocytes prompted a diagnosis of LAD-1.

The boy was isolated in a laminar air flow regimen, and HSCT was performed at 7 months of age from a matched unrelated donor. At 2 years after HSCT, $>99 \%$ of the patients' neutrophils and lymphocytes expressed normal levels of CD18. The patient is currently awaiting cardiac surgery to correct the subvalvular aortic stenosis.

Sequencing of the ITGB2 cDNA of the patient revealed a homozygous nonsense mutation in exon 3 (NM_000211.3: c.79A > T; NP_000202.2: p.Lys27X). The same mutation was detected in heterozygosis in the mother, but not in the father (Fig 1). Once paternity was confirmed, the presence of a gross deletion spanning the entire ITGB2 gene on the paternally derived allele was hypothesized.

Cytogenetic analysis on fibroblasts from a skin biopsy and performed using QFQ banding with $\geq 450$ band resolution revealed the presence of 1 normal chromosome 21 and a ring of the second 21 (Fig 2, A).
A

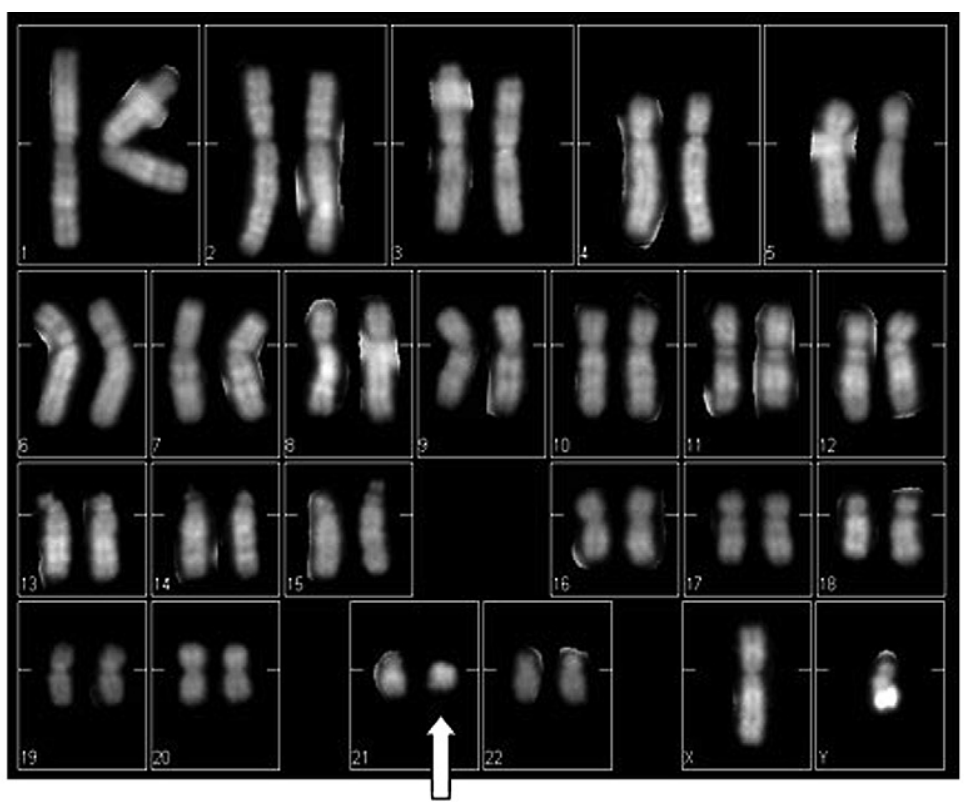

B

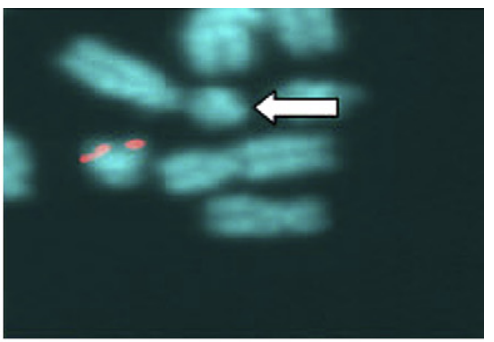

C

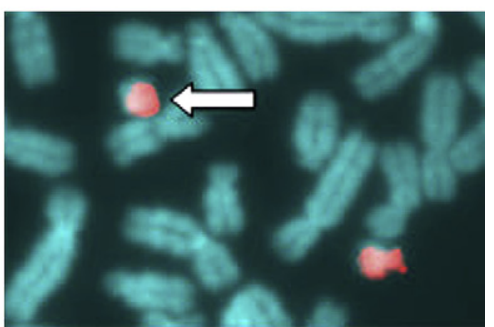

\section{Chromosome 21}

D

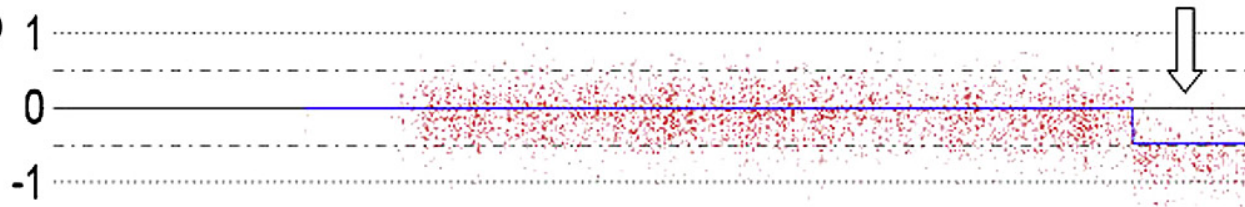

1

0

$-1$

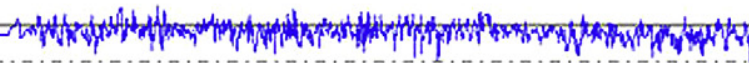

FIG 2. Cytogenetic and single nucleotide polymorphism array analyses. A, Patient's Q-banding (arrow). B, FISH painting of chromosome 21 shows signal on 2 chromosomes, including the ring chromosome (arrow). C, FISH using BAC clone RP11-15F6 reveals lack of signal on 1 of the 2 chromosomes 21 (arrow). D, Copy number state of chromosome 21 probes inferred by CNAG and reported as logarithm to the base 2 of the intensity ratio between proband and controls set. The arrow points to the deled region. 
Fluorescent in situ hybridization (FISH) with painting probe (WCP) of chromosome 21 confirmed that the ring was derived from chromosome 21, whereas FISH performed by using a $21 \mathrm{q}-$ specific telomere probe and BAC clone RP11-15F6, containing the ITGB2 sequence, showed a complete lack of signals on the $\mathrm{r}(21)$ chromosome (Fig 2, $B$ and $C$ ). On the basis of these findings, we determined the karyotype to be $46, \mathrm{XY}$, ish $\mathrm{r}(21)$ (WCP+, 21qtel-, RP11-15F6-). ${ }^{9}$

Conventional chromosomal and FISH analysis with the same probes revealed normal results in both parents.

The Whole Genome Analysis of the trio with the Affymetrix GeneChip Human Mapping 250 K NspI array (Affymetrix, Santa Clara, Calif) confirmed the presence in the proband of a heterozygous de novo deletion in the chromosome region 21q22.3-qter (Fig 2,D). According to CNAT4 software (Affymetrix), the deletion breakpoint mapped between SNP_A-2020087 (42,346,671 bp) and SNP_A-4209155 (42,365,654 bp), whereas, according to CNAG software (Affymetrix), the breakpoint was included between 42,374,607 bp and 42,389,446 bp. The deletion spanned $4.6 \mathrm{Mb}$ and, besides the ITGB2 gene, included 69 additional genes, among which were the $A D A R B 1$ gene encoding for the enzyme responsible for pre-mRNA editing of the glutamate receptor subunit $\mathrm{B}$, and COL18A, COL6A, and COL6A2 genes encoding for members of the collagen superfamily.

These results confirm that most often different pathologies occurring in the same person can be related to 1 common cause, and emphasize the importance of including cytogenetic analysis in patients with complex clinical phenotypes.

The boy we have reported, besides the typical features of $\mathrm{r}(21)$, also had the characteristic findings of LAD-1. Typically, the loss of 1 allele of ITGB2 does not lead to a clinical immunodeficiency because this disease is inherited as an autosomal-recessive trait; however, in our patient, the LAD-1 phenotype was disclosed by an ITGB2 nonsense mutation that he inherited from the heterozygous mother. CD18 is expressed on blood leukocytes, and hence, donor stem cell engraftment leads to correction of the LAD-1 phenotype. Although LAD-1 can be cured by HSCT, this is not the case for the other $\mathrm{r}(21)$-associated clinical features. Indeed, at 18 months of age, although he has no evidence of immune deficiency, he remains beneath the third percentile for height, weight, and cranial circumference, and also shows signs of neurodevelopmental delay.

We thank the Sanger Center (Cambridge, United Kingdom), which provided the BAC clone for FISH experiments, and Mrs Goffi and Mrs Rizzini for their technical support of this work.

\section{Maurilia Fiorini, $P h D^{a *}$ Giovanna Piovani, $P h D^{d *}$ Richard F. Schumacher, $M D^{b}$ Chiara Magri, $P h D^{d}$ Valeria Bertini ${ }^{d}$ Evelina Mazzolari, $M D^{b}$ Lucia Notarangelo, $M D^{b}$ Luigi D. Notarangelo, $M D^{c, e}$ Sergio Barlati, $P h D^{d}$}

From athe "Angelo Nocivelli" Institute for Molecular Medicine and bivisione di Emato-Oncologia Pediatrica and ${ }^{\mathrm{c}}$ Clinica Pediatrica, Ospedale dei Bambini, Spedali Civili, and ${ }^{\mathrm{d}}$ Biology and Genetics, Department Biomedical Sciences and Biotechnology, University of Brescia, Italy; and ${ }^{\mathrm{e}}$ the Division of Immunology and Manton Center for Orphan Disease Research, Children's Hospital, Harvard Medical School, Boston, Mass. E-mail: barlati@med.unibs.it

*These authors contributed equally to this work.
Supported by Centro di Studio e ricerca sulle malattie Ereditarie and MIUR (to S.B.) and the Manton Foundation (to L.N.).

Disclosure of potential conflict of interest: L. Notarangelo has received research support from the National Institutes of Health and the Manton Foundation. The rest of the authors have declared that they have no conflict of interest.

\section{REFERENCES}

1. Marlin SD, Morton CC, Anderson DC, Springer TA. LFA-1 immunodeficiency disease: definition of the genetic defect and chromosomal mapping of alpha and beta subunits of the lymphocyte function-associated antigen 1 (LFA-1) by complementation in hybrid cells. J Exp Med 1986;164:855-67.

2. Corbi AL, Larson RS, Kishimoto TK, Springer TA, Morton CC. Chromosomal location of the genes encoding the leukocyte adhesion receptors LFA-1, Mac-1 and p150,95: identification of a gene cluster involved in cell adhesion. J Exp Med 1988;167: 1597-607.

3. Solomon E, Palmer RW, Hing S, Law SK. Regional localization of CD18, the betasubunit of the cell surface adhesion molecule LFA-1, on human chromosome 21 by in situ hybridization. Ann Hum Genet 1988;52:123-8.

4. Fischer A, Lisowska-Grospierre B, Anderson DC, Springer TA. Leukocyte adhesion deficiency: molecular basis and functional consequences. Immunodefic Rev 1988;1:39-54.

5. Arnaout MA. Leukocyte adhesion molecules deficiency: its structural basis, pathophysiology and implications for modulating the inflammatory response. Immunol Rev 1990;114:145-80.

6. Lejeune J, Berger R, Rethore MO, Archambault L, Jerome H, Thieffry S, et al. Partial monosomy for a small acrocentric chromosome. C R Hebd Seances Acad Sci 1964;259:4187-90.

7. Ieshima A, Ogasawara N, Yamamoto Y, Kuroki Y. A case of r(21) with stigmata of atypical Down syndrome. Hum Genet 1980;55:65-9.

8. Ferrante E, Vignetti P, Antonelli M, Bruni L, Bertasi S, Chessa L. Partial monosomy for a 21 chromosome. Report of a new case of $\mathrm{r}(21)$ and review of the literature. Helv Paediatr Acta 1983;38:73-80.

9. Shaffer LG, Tommerup NISCN. an international system for human cytogenetic nomenclature. Basel: S. Karger; 2005.

Available online October 28, 2009 doi:10.1016/j.jaci.2009.07.058

\section{Placental transfer of allergen-specific IgG but not IgE from a specific immunotherapy- treated mother}

\section{To the Editor:}

Several reports suggest that allergic sensitization can be influenced already in utero. ${ }^{1}$ Some studies indicate that allergic sensitization can occur in utero by transfer of allergens via the placenta to the child, ${ }^{2,3}$ whereas others have argued against this possibility and suggest that allergen-specific IgE in cord blood does not reflect intrauterine sensitization but rather is the result of a transfer of maternal IgE to the fetus. ${ }^{4}$ However, whether IgE and in particular allergen-specific IgE can be transferred via the placenta to the child represents a controversial issue. The presence of allergen-specific IgE antibodies in children with specificities similar to those of their mothers has been reported in 2 studies, ${ }^{4,5}$ whereas others suggested that the placenta represents a barrier for IgE antibodies. 6

We had the opportunity to study the antibody reactivity profiles in serum samples from a mother with birch and grass pollen allergy (IgE levels before specific immunotherapy: 18.50 kilo units [kU]/L total $\mathrm{IgE}, 1.8 \mathrm{kUA} / \mathrm{L}$ recombinant $[\mathrm{r}]$ Bet $\mathrm{v} 1,2.48 \mathrm{kUA} / \mathrm{L} \mathrm{rPhl} \mathrm{p}$ $1+\mathrm{rPhl} p$ 5) who had received subcutaneous injection immunotherapy with birch and grass pollen extracts (Alutard SQ; ALKAbelló, Hørsholm, Denmark) for 2 years before she became pregnant and delivered her baby as well as in the corresponding cord blood sample of her child using an array of purified allergen molecules. To the best of our knowledge, this is the first detailed analysis of allergen-specific $\operatorname{IgE}$ (Fig 1 ), $\mathrm{IgG}_{1}$, and $\mathrm{IgG}_{4}$ antibodies (Fig 2) to purified allergen molecules from several different 